\title{
CARACTERIZACIÓN DEL CRECIMIENTO Y DESARROLLO DEL MAÍZ EN CONDICIONES DE NO LABOREO EN LA MANCHA ORIENTAL
}

\author{
Barroso Costa, F. R. ${ }^{1}$ Ortega Álvarez, J. F. ${ }^{2}$ Ballesteros González, R. ${ }^{3}$
}

1 Ingeniero Agrónomo, Estudiante de doctorado, Centro Regional de Estudios del Agua (CREA), Universidad de Castilla-La Mancha, Ctra. de Las Peñas km. 3.4, 02071 Albacete, fellyperodrigo.barrosocosta@alu.uclm.es.

${ }^{2}$ Profesor Titular, Centro Regional de Estudios del Agua (CREA), Universidad de Castilla-La Mancha, Ctra. de Las Peñas km. 3.4, 02071 Albacete, jose.ortega@uclm.es.

${ }_{3}$ Personal Investigador, Centro Regional de Estudios del Agua (CREA), Universidad de Castilla-La Mancha, Ctra. de Las Peñas km. 3.4, 02071 Albacete, rocio.ballesteros@uclm.es.

\section{Resumen}

La biomasa es se define como la materia orgánica, viva o muerta, que se produce por un cultivo. Por medio de la metodología de los grados-día acumulados (GDA), se puede cuantificar la energía acumulada por el cultivo durante su ciclo. El objetivo de este trabajo es estudiar los principales parámetros que caracterizan el cultivo del maíz, en una parcela comercial y en condiciones de no laboreo, en una zona semiárida localizada en La Mancha Oriental. Se realizó un seguimiento fenológico durante todo el ciclo en una parcela comercial de maíz grano durante la campaña de riego del año 2015. Se recogieron muestras de plantas durante todo el ciclo del maíz. Se registraron los cambios fenológicos del cultivo siguiendo para ello la escala $\mathrm{BBCH}$. Con los datos obtenidos en campo y en el laboratorio, los resultados obtenidos de Índice de Área Foliar (IAF) frente a días después de emergencia (DDE), Materia Seca (MS) frente a GDA, entre otros, fueron sometidos a un análisis estadístico descriptivo. Los resultados estadísticos indican que la MS frente a DDE presentó mejor ajuste en el modelo logístico, con un coeficiente de determinación $\left(R^{2}\right)$ de 0,96 y raíz del error cuadrático medio $(\mathrm{RMSE})=248,55 \mathrm{~g} \mathrm{~m}^{-2}$. En relación al IAF, las dos metodologías (DDE y GDA) presentaron mejor ajuste en el modelo polinomial cuadrático, con $R^{2}=0,85$ y 0,88 , y RMSE $=0,88 \mathrm{~m}^{2}$ planta $\mathrm{m}^{-2}$ suelo y $0,78 \mathrm{~m}^{2}$ planta $\mathrm{m}^{-2}$ suelo, respectivamente. Se concluye que la MS y el IAF presentan comportamientos distintos en el cultivo del maíz. Con los valores de los GDA calculados, fue posible definir la duración de cada etapa del desarrollo fisiológico del maíz.

\section{1 - Introducción}

El maíz es un cultivo de gran importancia en la región de Castilla-La Mancha. El tipo más tradicional es el maíz grano, con variedades del ciclo 600 a 700, que en la región, tiene una producción correspondiente a un 9\% de la producción nacional. Estas variedades se caracterizan por una alta producción de biomasa seca, variando de 21 a $27 \mathrm{t} \mathrm{ha}^{-1}$ (Lloveras, Martínez, \& Santiveri, 2014).

La biomasa es, por definición, toda la materia orgánica, viva o muerta, producida por un cultivo, esté sobre el suelo o por debajo de él. La materia seca (MS) se refiere a lo que resta de un material tras extraer todo su contenido de agua posible, a través del secado hecho en laboratorio, en una estufa a unos $70^{\circ} \mathrm{C}$. 
Por medio de la metodología de los grados-día acumulados (GDA), es posible cuantificar la energía acumulada por la planta en cada etapa de su desarrollo. El grado-día consiste en la cantidad de energía calorífica que la planta consigue acumular diariamente (Ballesteros, 2014; Íñiguez-Covarrubias, Ojeda-Bustamante, \& Sifuentes-Ibarra, 2014). La aplicación de la metodología de los GDA permite estudiar y conocer en profundidad el desarrollo del cultivo, planificando así un adecuado calendario de riegos (Akmal, Asim, \& Gilbert, 2014).

Los cultivos anuales, como el caso del maíz, dividen su ciclo en cuatro etapas de desarrollo fenológico, con cada etapa teniendo duración distinta y variable. La etapa I es de establecimiento del cultivo, en la cual hay un crecimiento más lento del cultivo. La etapa II presenta rápido crecimiento, con mayor desarrollo de las hojas y del tallo. La etapa III empieza con el inicio de la floración. En esa etapa la planta dirige sus reservas en el desarrollo de la mazorca y llenado de los granos. También en esta etapa, el cultivo alcanza su máximo desarrollo y la máxima cubierta del suelo. La última etapa (IV) presenta una disminución de los valores de algunos parámetros fisiológicos, como la MS y del Índice de Área Foliar (IAF), ya que el cultivo inicia la senescencia (Allen, Pereira, Raes, \& Smith, 2006).

Una de las prácticas conservacionistas que más se utiliza hoy es el cultivo de no laboreo. En cual consiste en trabajar lo mínimo posible la tierra, haciendo la siembra directa del cultivo en el rastrojo de la campaña anterior. En esta práctica, el control de las malas hierbas se consigue con la aplicación de herbicidas. Además el rastrojo protege el suelo contra la erosión (Cid, Carmona, García, \& Mcpherson, 2014).

El objetivo de este trabajo es estudiar los principales parámetros que caracterizan el cultivo del maíz, en una parcela comercial y en condiciones de no laboreo, en una zona semiárida localizada en La Mancha Oriental. Además, se propone estudiar el ciclo del maíz mediante la metodología de los GDA y de los datos recogidos en campo.

\section{2 - Material e métodos}

Se realizó un seguimiento fenológico durante todo el ciclo en una parcela comercial de maíz grano, variedad P-1114, en una finca ubicada en Tarazona de La Mancha (Albacete) en la Unidad Hidrogeológica (U.H.) 08.29. (Mancha Oriental), durante la campaña de riego del año 2015. La región se caracteriza por ser de clima semiárido, con pluviometría media anual de $393,22 \mathrm{~mm}$.

Se registraron los cambios fenológicos del cultivo siguiendo para ello la escala BBCH (Weber \& Bleiholder, 1990) de forma semanal, lo que permitió una adecuada planificación de los muestreos en campo. Considerando los cambios más representativos en la fenología del cultivo se realizaron once de estos muestreos. La parcela se dividió en cuatro zonas distintas, considerando distintos tipos de suelo y topografía. Los puntos de muestreo fueron escogidos de forma aleatoria, muestreando una subparcela de cada una de las zonas anteriormente descritas en cada día de muestreo. El área de muestreo fue de $1 \times 0,7 \mathrm{~m}$. En cada muestreo, se recogieron 6 plantas por zona, siendo 24 plantas muestreadas cada día. También se midió la densidad de plantas de cada subparcela, además de otros parámetros como altura total de la planta y longitud de distintos órganos.

En laboratorio, fueron separados los distintos órganos de la planta. Después, se obtuvo el área foliar de cada planta utilizando el medidor de área foliar LICOR, modelo LI3100C. Posteriormente, se pesó cada uno de los órganos de las plantas por separado, y por último, éstos se introdujeron en una estufa a una temperatura constante de $70^{\circ} \mathrm{C}$ hasta alcanzar un peso constante, determinando así la biomasa seca.

Con los datos obtenidos en campo y en el laboratorio, se obtuvo el IAF, la MS de cada parte de la planta y la MS total. En paralelo con los resultados obtenidos en campo, se calcularon los GDA a través de la metodología del seno doble (Allen, 1976), durante todo el ciclo del maíz, posibilitando hacer una comparación con el desarrollo del cultivo en relación a los días después de emergencia (DDE). De esta forma, los resultados obtenidos de IAF frente a DDE, IAF frente a GDA, MS total frente a DDE, MS total frente a GDA, MS total 
frente a altura de plantas y altura de plantas frente a DDE, fueron sometidos a un análisis estadístico descriptivo. Las ecuaciones y gráficas que mejor se ajustaron a los modelos fueron estimadas utilizando el software MATLAB R2013b.

\section{3 - Resultados y discusión}

Los resultados estadísticos indican que la MS frente a DDE (Figura 1) presentó mejor ajuste en el modelo logístico, con un $\mathrm{R}^{2}$ de 0,96 y $\mathrm{RMSE}=248,6 \mathrm{~g} \mathrm{~m}^{-2}$. Eso indica que el cultivo del maíz tiene una producción de MS lenta en la etapa I (1 a $47 \mathrm{DDE}$ ), aumentando su crecimiento en la etapa II (48 a 75 DDE), hasta que alcanza su máxima producción de MS en la etapa III (76 a 154 DDE), alcanzando una producción máxima puntual de MS total de hasta $31.200 \mathrm{~kg} \mathrm{ha}^{-1}$ en una de las zonas de muestreo. A partir de entonces hay una pequeña reducción no relevante en la MS en la etapa IV (a partir de 155 DDE), debido al proceso de senescencia del cultivo.

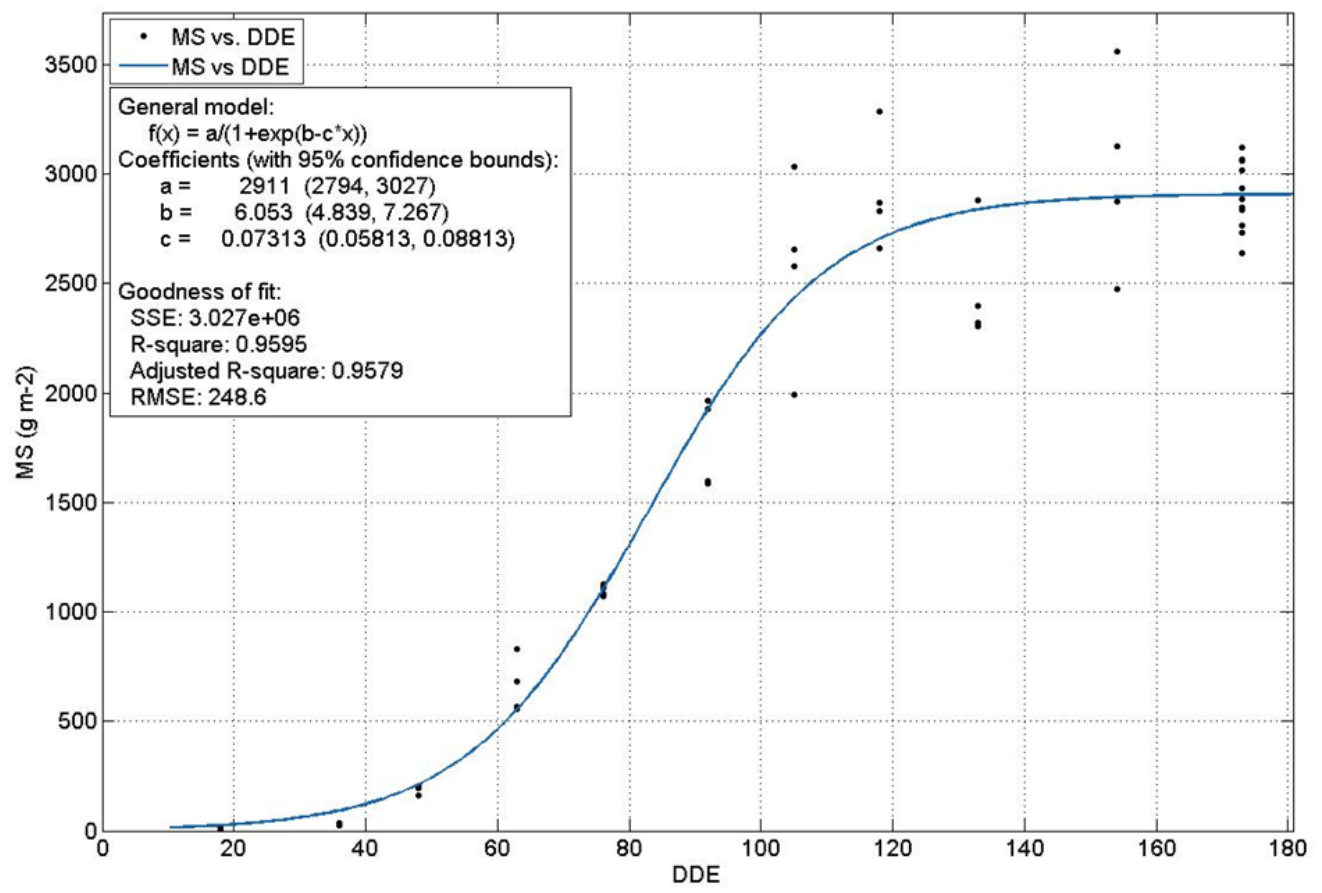

Figura 1. MS frente a DDE en maíz.

Resultado similar fue encontrado por Junior et al., (2014), que, estudiando el maíz en condiciones semiáridas en Alagoas, Brasil, describió cómo el maíz, en el inicio del ciclo, prioriza la acumulación de fotoasimilados para el crecimiento de sus hojas y tallo, y que, Ilegando a la etapa III, dirige su reserva para el desarrollo de la mazorca y posterior llenado de granos. Singer, Meek, Sauer, Prueger, \& Hatfield (2011), estudiando la producción de biomasa en maíz, encontraron valores de MS similares a los de este estudio, con el crecimiento inicial un poco más lento, una rápida producción de biomasa en la etapa de crecimiento y alcanzando su máxima MS media en la etapa productiva, con valores menores que los de este estudio ( $2.300 \mathrm{~g} \mathrm{~m}^{-2}$ frente a $3.000 \mathrm{~g} \mathrm{~m}^{-2}$ de este estudio).

La MS frente a GDA (Figura 2) presentó un mejor ajuste con el modelo logístico, con $R^{2}=0,96$ y RMSE $=247,6 \mathrm{~g} \mathrm{~m}^{-2}$. En esa gráfica se puede ver que la MS, cuando relacionada con los GDA, presenta un crecimiento más lento en la primera etapa (hasta 562,12 GDA), en la etapa II presenta crecimiento exponencial (562,13 a 1.015,84 GDA), luego estabiliza el crecimiento en la etapa III, alcanzando su mayor desarrollo (1.015,85 a 2.262,85 GDA) y empieza la senescencia y disminuye un poco su MS en la etapa IV (desde 2.262,86 GDA). 


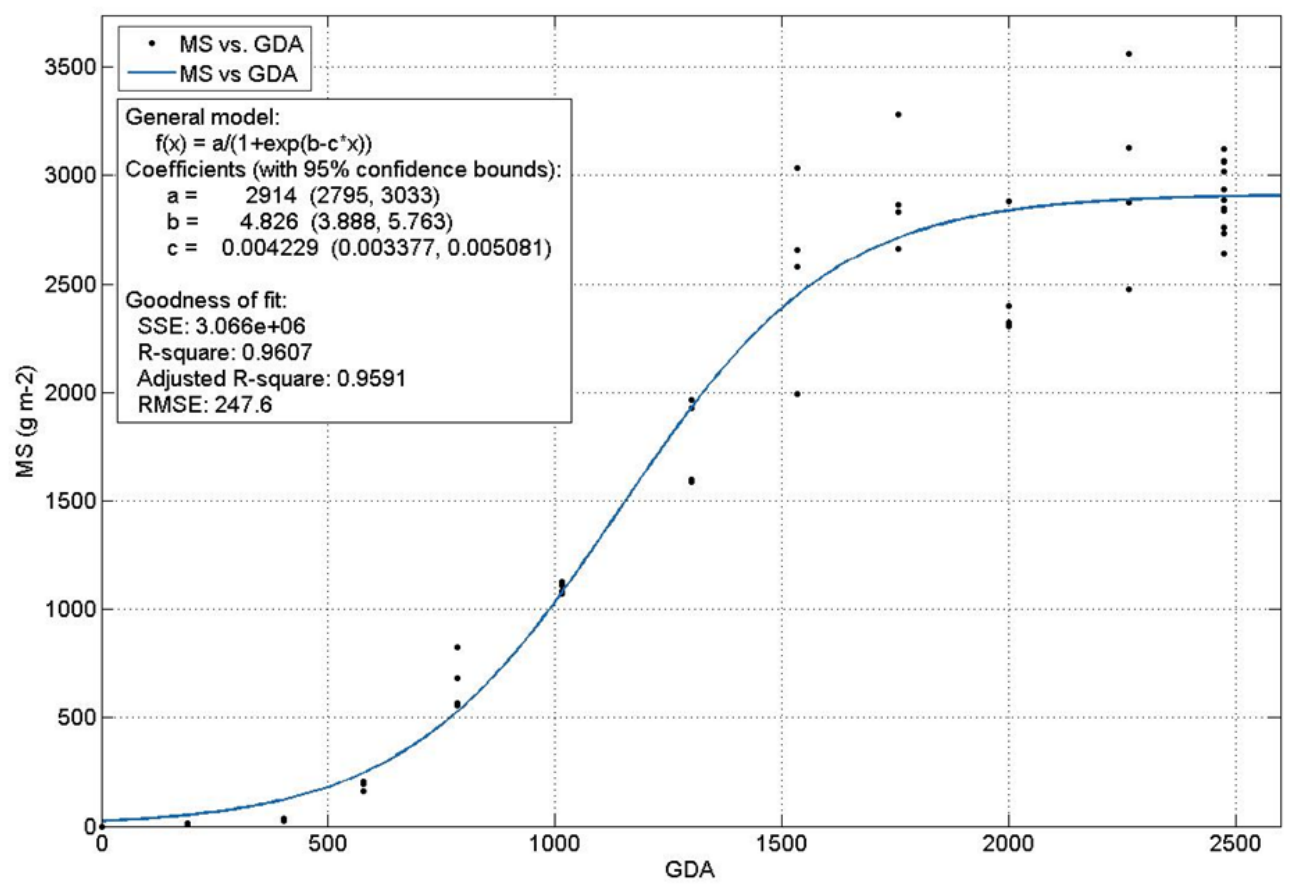

Figura 2. MS frente a GDA en maíz.

En relación al IAF, se nota el mismo comportamiento en las dos metodologías (DDE y GDA) (Figuras 3 y 4), ya que ambos presentaron mejor ajuste en el modelo polinomial cuadrático, con $R^{2}=0,85$ y 0,88 , y RMSE $=0,88 \mathrm{~m}^{2}$ planta $\mathrm{m}^{-2}$ suelo y $0,78 \mathrm{~m}^{2}$ planta $\mathrm{m}^{-2}$ suelo, respectivamente.

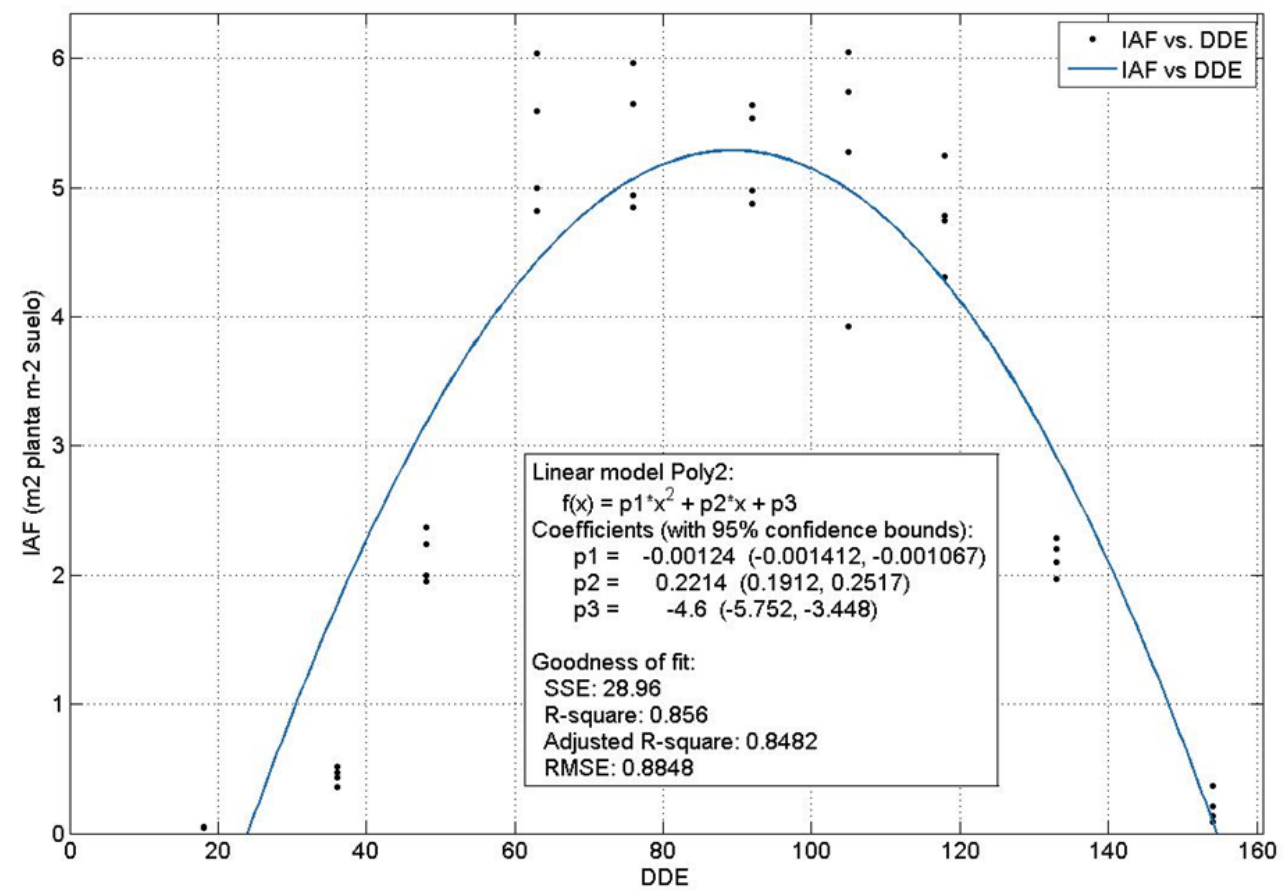

Figura 3. IAF frente a DDE en maíz.

Eso indica que el IAF aumenta de acuerdo con el desarrollo de la planta de maíz, alcanzando su máximo valor en el final de la etapa II, manteniendo los valores similares hasta el final de la etapa III, cuando empieza a decaer, debido a la senescencia del cultivo. 


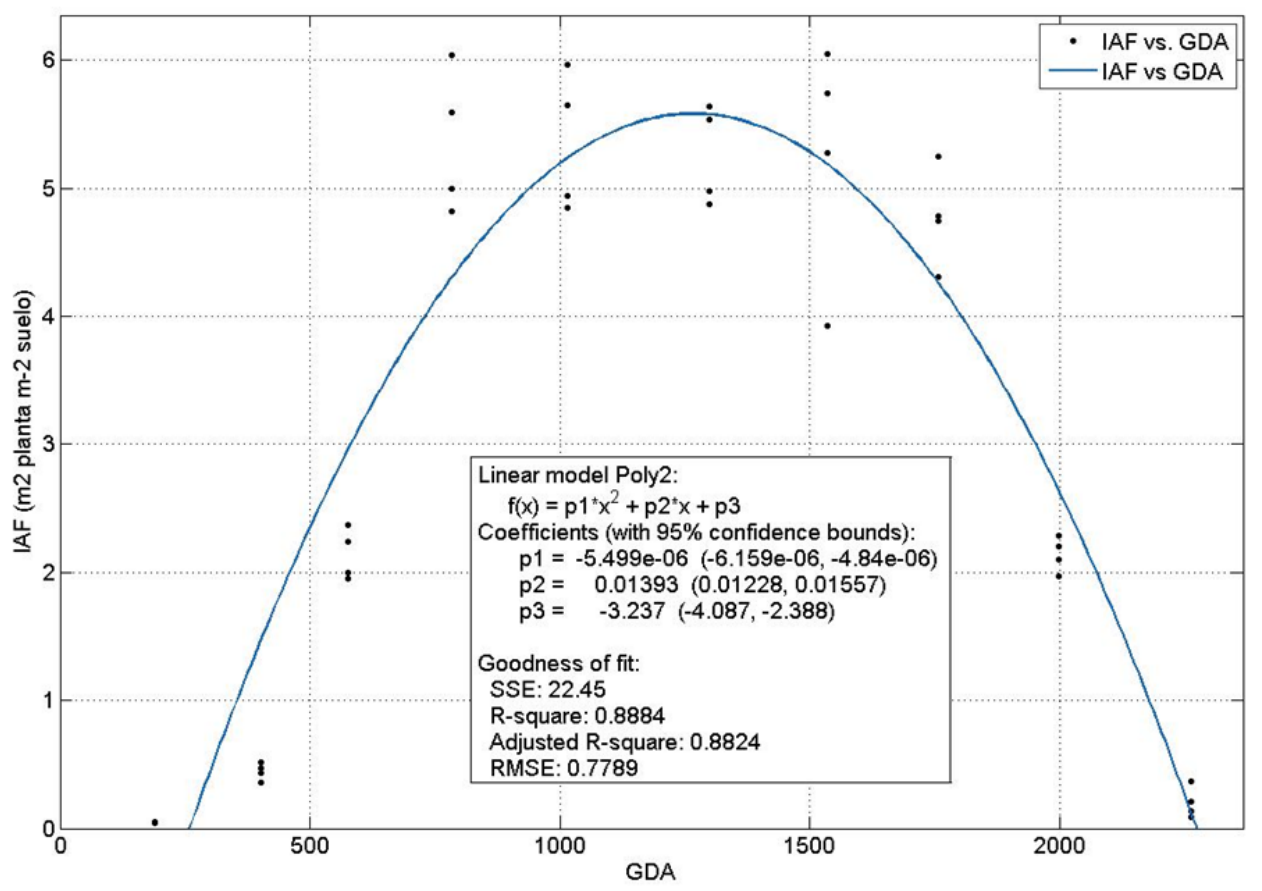

Figura 4. IAF frente a GDA en maíz.

Autores como Zegada-Lizarazu, Zatta, \& Monti (2012), encontraron valores de IAF en maíz semejantes a los encontrados en este estudio, ya que el valor de IAF estuvo cerca de $6 \mathrm{~m}^{2}$ planta $\mathrm{m}^{-2}$ suelo, en la etapa III del ciclo del maíz, reduciendo su valor al final de esta etapa, coincidiendo con el inicio de la senescencia del maíz. Lo mismo ocurrió en el trabajo de Quanqi, Yuhai, Xunbo, Songlie, \& Changcheng (2012), que obtuvo un comportamiento del IAF en el ciclo del maíz muy similar al de este estudio, obteniendo valores cercanos a los $6 \mathrm{~m}^{2}$ planta $\mathrm{m}^{-2}$ suelo en la etapa III, y luego disminuyendo los valores en la fase final del ciclo del maíz.

La MS frente a altura de plantas (Figura 5) presentó mejor ajuste en el modelo de Gompertz, con valores de $\mathrm{R}^{2}=0,87$ y RMSE $=434 \mathrm{~g} \mathrm{~m}^{-2}$. A partir del modelo se nota que, en el cultivo del maíz, a medida en que aumenta la altura de las plantas, el cultivo aumenta su producción de MS. Normalmente, las plantas más altas producen más MS, debido a que sus tallos almacenan sus reservas. Otra razón es que plantas más altas interceptan más radiación solar, posibilitando así un mejor desarrollo de la mazorca. 


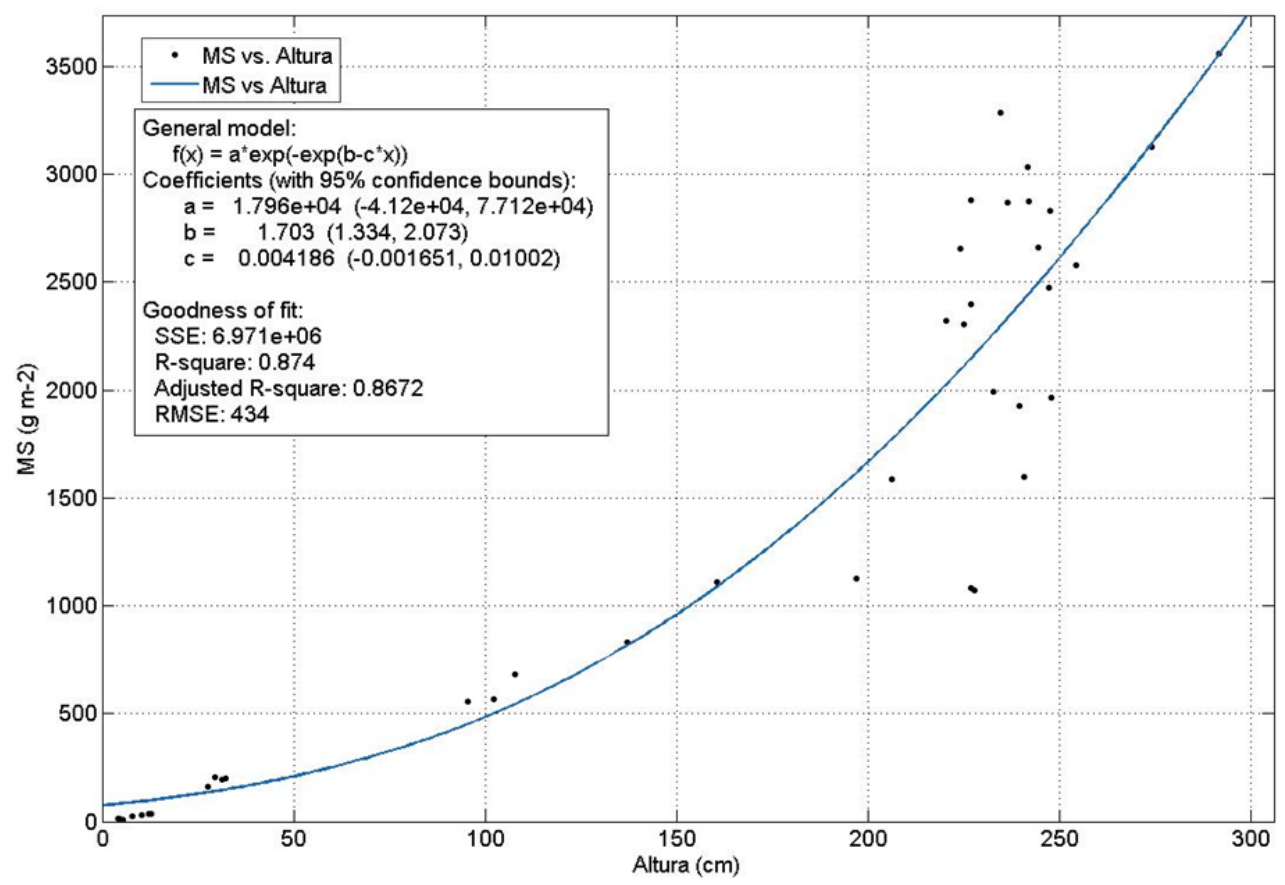

Figura 5. MS frente a Altura de plantas de maíz.

Schittenhelm \& Schroetter (2014), comparando la MS de plantas de maíz con su altura, encontraron que las plantas con altura media de $280 \mathrm{~cm}$ tenían una producción de MS de $1.910 \mathrm{~g} \mathrm{~m}^{-2}$, en cuanto que en este estudio, las plantas con altura media de $280 \mathrm{~cm}$ tuvieron una producción de MS de $3.279,37 \mathrm{~g} \mathrm{~m}^{-2}$.

En relación a la altura de plantas frente a DDE (Figura 6), se observa que la altura de las plantas tiene un aumento muy lento en la etapa I, aumenta exponencialmente en la etapa II, alcanza su máximo en la etapa III, y se mantiene constante hasta el fin del ciclo. El modelo que mejor se ajustó a ese parámetro fue el logístico, con $R^{2}=0,97$ y $R M S E=$ $17,01 \mathrm{~cm}$.

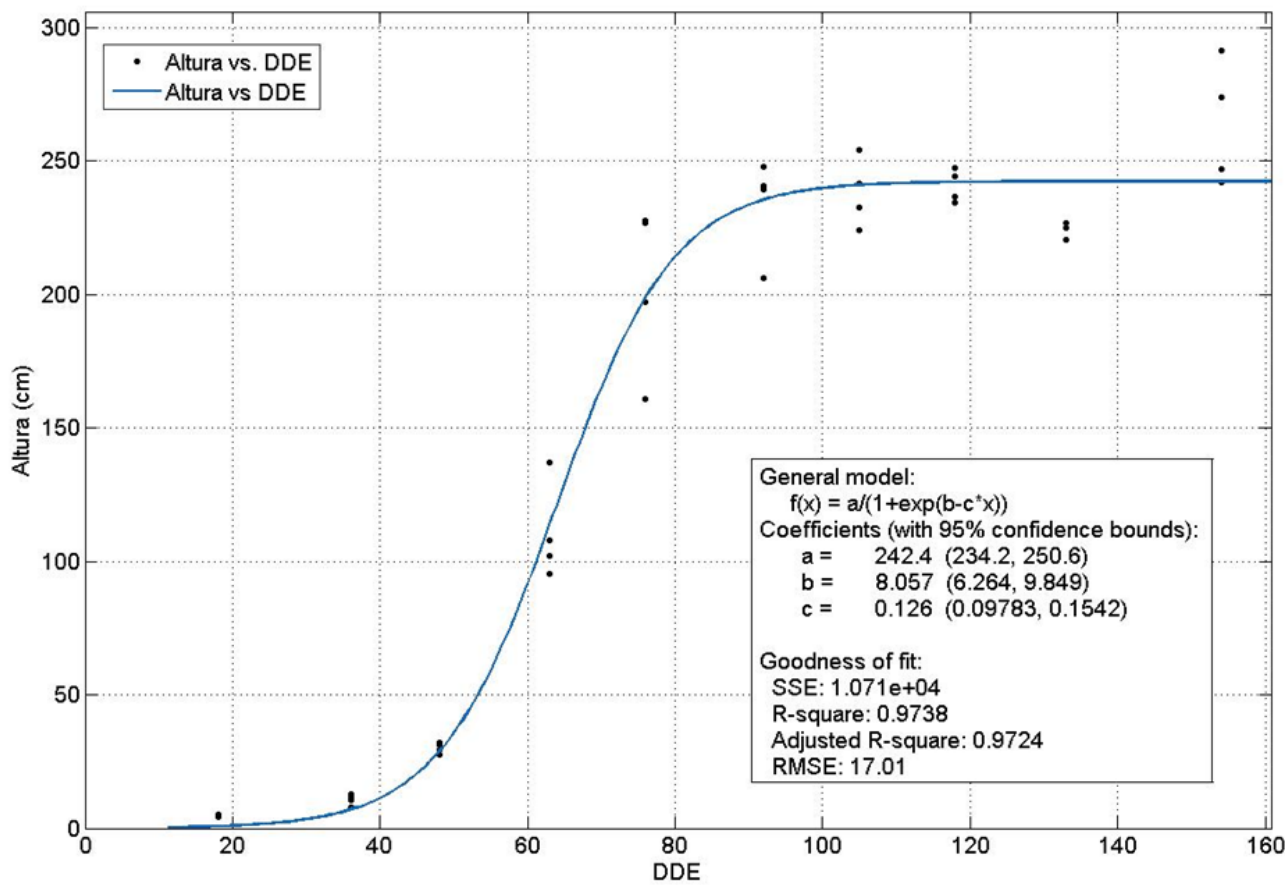

Figura 6. Altura de plantas frente a DDE en maíz. 
Autores como Liu, Chen, Liu, Wen, \& Liao (2016), obtuvieron valores de altura de planta muy similares a los de este estudio durante todo su ciclo, con alturas medias en la etapa III cerca de los $250 \mathrm{~cm}$.

\section{4 - Conclusiones y recomendaciones}

Con los valores de los GDA calculados, fue posible definir la duración de cada etapa del desarrollo fisiológico del maíz, lo que permite hacer una planificación del ciclo del cultivo, en lo que corresponde a la aplicación de agua, fertilizantes y operaciones culturales en el cultivo de maíz.

La MS y el IAF presentan comportamientos distintos en el cultivo del maíz, ya que el cultivo sólo alcanza su máxima MS al final de la etapa III, en cuanto el IAF máximo se da en el final de la etapa II y se mantiene hasta el fin de la etapa III. En relación al final del ciclo, la MS decrece muy poco en relación a su máximo valor alcanzado, en cuanto que el IAF se reduce mucho en la última etapa.

\section{5 - Agradecimientos}

Agradezco al Proyecto IBrasil - Erasmus Mundus por financiar mis estudios de doctorado en la Universidad de Castilla-La Mancha.

\section{6 - Referencias}

Akmal, M., Asim, M., \& Gilbert, M. (2014). Influence of seasonal variation on radiation use efficiency and crop growth of maize planted at various densities and nitrogen rates. Pakistan Journal of Agricultural Science, 51(4), 835-846.

Allen, J. C. (1976). A modified sine wave method for calculating degree days. Environmental Entomology, 5(3), 388-396.

Allen, R. G., Pereira, L. S., Raes, D., \& Smith, M. (2006). Evapotranspiración del cultivo Guías para la determinación de los requerimientos de agua de los cultivos. Manual FAO-56. Food and Agriculture Organization (FAO). Rome.

Ballesteros, R. (2014). Predicción de evapotranspiración de referencia y empleo de modelos de tiempo térmico junto a información geomática en la caracterización de cultivos para la gestión del regadío en la Mancha Oriental. Tesis Doctoral. Escuela Tecnica Superior de Ingenieros Agrónomos y de Montes. Universidad de Castilla-La Mancha, Albacete, España.

Cid, P., Carmona, I., García, R. C., \& Mcpherson, H. G. (2014). Claves para la viabilidad de la agricultura de conservación en la producción de maíz en regadío. Vida Rural, 382, 32-37.

Íñiguez-Covarrubias, M., Ojeda-Bustamante, W., \& Sifuentes-Ibarra, C. D. E. (2014). Análisis de cuatro variables del período de lluvias asociadas al cultivo maíz de temporal. Revista Mexicana de Ciencias Agrícolas, 5, 101-114.

Junior, R. A. F., Souza, J. L. De, Teodoro, I., Lyra, G. B., Souza, R. C. De, \& Neto, R. A. D. A. (2014). Eficiência do uso da radiação em cultivos de milho em Alagoas Radiation use efficiency in corn crop in Alagoas. Revista Brasileira de Engenharia Agrícola e Ambiental, 18(3), 322-328.

Liu, Q., Chen, Y., Liu, Y., Wen, X., \& Liao, Y. (2016). Coupling effects of plastic fi Im mulching and urea types on water use efficiency and grain yield of maize in the Loess Plateau, China. Soil and Tillage Research, 157, 1-10.

Lloveras, J., Martínez, E., \& Santiveri, F. (2014). Influencia de la fecha de siembra en el maíz en regadío del valle del Ebro. Vida Rural, 374, 36-40.

Quanqi, L., Yuhai, C., Xunbo, Z., Songlie, Y., \& Changcheng, G. (2012). Effect of irrigation to winter wheat on the radiation use efficiency and yield of summer maize in a double cropping system. The Scientific World Journal, 476272. 
Schittenhelm, S., \& Schroetter, S. (2014). Comparison of Drought Tolerance of Maize, Sweet Sorghum and Sorghum-Sudangrass Hybrids. Journal of Agronomy and Crop Science, 200(1), 46-53.

Singer, J. W., Meek, D. W., Sauer, T. J., Prueger, J. H., \& Hatfield, J. L. (2011). Variability of light interception and radiation use efficiency in maize and soybean. Field Crops Research, 121(1), 147-152.

Weber, E., Bleiholder, H. (1990). Erläuterungen zu den BBCH-Dezimal-Codes für die Entwicklungsstadien von Mais, Raps, Faba-Bohne, Sonnenblume und Erbse - mit Abbildungen. Gesunde Pflanzen, 42, 308-321.

Zegada-Lizarazu, W., Zatta, A., \& Monti, A. (2012). Water uptake efficiency and above and belowground biomass development of sweet sorghum and maize under different water regimes. Plant and Soil, 351(1-2), 47-60. 